\title{
A revised model of platelet aggregation
}

Suhasini Kulkarni, ${ }^{1}$ Sacha M. Dopheide, ${ }^{1}$ Cindy L. Yap, ${ }^{1}$ Catherine Ravanat, ${ }^{2}$ Monique Freund, ${ }^{2}$ Pierre Mangin, ${ }^{2}$ Kathryn A. Heel, ${ }^{1}$ Alison Street, ${ }^{3}$ Ian S. Harper, ${ }^{1}$ Francois Lanza, ${ }^{2}$ and Shaun P. Jackson ${ }^{1,4}$

\author{
${ }^{1}$ Australian Centre for Blood Diseases, Department of Medicine, Monash Medical School, Box Hill Hospital, Box Hill, \\ Victoria 3128, Australia \\ ${ }^{2}$ Etablissement de Transfusion Sanguine, Institut National de la Sante et de la Recherche Medicale Unite 311, \\ 67065 Strasbourg Cedex, France \\ ${ }^{3}$ Department of Pathology, Alfred Hospital, Prahran, Victoria 3181, Australia \\ ${ }^{4}$ Department of Pathology, Box Hill Hospital, Box Hill, Victoria 3128, Australia
}

Address correspondence to: Shaun P. Jackson, Australian Centre for Blood Diseases, Department of Medicine, Monash Medical School, Box Hill Hospital, Arnold Street, Box Hill, Victoria 3128, Australia.

Phone: 61-3-9895-0311; Fax: 61-3-9895-0332; E-mail: Shaun.Jackson@med.monash.edu.au.

Received for publication June 11, 1999, and accepted in revised form January 11, 2000.

In this study we have examined the mechanism of platelet aggregation under physiological flow conditions using an in vitro flow-based platelet aggregation assay and an in vivo rat thrombosis model. Our studies demonstrate an unexpected complexity to the platelet aggregation process in which platelets in flowing blood continuously tether, translocate, and/or detach from the luminal surface of a growing platelet thrombus at both arterial and venous shear rates. Studies of platelets congenitally deficient in von Willebrand factor (vWf) or integrin $\alpha_{\mathrm{IIb}} \beta_{3}$ demonstrated a key role for platelet $\mathrm{vWf}$ in mediating platelet tethering and translocation, whereas integrin $\alpha_{\text {II }} \beta_{3}$ mediated cell arrest. Platelet aggregation under flow appears to be a multistep process involving: (a) exposure of vWf on the surface of immobilized platelets; (b) a reversible phase of platelet aggregation mediated by the binding of GPIb $\alpha$ on the surface of free-flowing platelets to vWf on the surface of immobilized platelets; and (c) an irreversible phase of aggregation dependent on integrin $\alpha_{\mathrm{II}} \beta_{3}$. Studies of platelet thrombus formation in vivo demonstrate that this multistep adhesion mechanism is indispensable for platelet aggregation in arterioles and also appears to promote platelet aggregate formation in venules. Together, our studies demonstrate an important role for platelet $\mathrm{vWf}$ in initiating the platelet aggregation process under flow and challenge the currently accepted view that the vWf-GPIb $\alpha$ interaction is exclusively involved in initiating platelet aggregation at elevated shear rates.

J. Clin. Invest. 105:783-791 (2000).

\section{Introduction}

Platelet aggregation at sites of vascular injury is essential for the formation of the primary hemostatic plug and also for the development of pathological thrombi at sites of atherosclerotic plaque rupture. The initial contact of platelets with the injured vessel wall (platelet adhesion) is a complex process involving multiple adhesive substrates (von Willebrand factor [vWf], collagen) and receptors on the platelet surface (GPIb/V/IX, integrins $\alpha_{\mathrm{II}} \beta_{3}$ and $\alpha_{2} \beta_{1}$ ) (1). The interaction between matrix-bound vWf and GPIb $\alpha$ on the platelet surface serves primarily to tether platelets to the area of vascular injury $(2,3)$, particularly under conditions of high shear stress, as a prerequisite step for integrin-mediated cell arrest (4). Whereas the molecular events underlying platelet adhesion under different shear conditions have been well delineated, the mechanism(s) by which platelets in freeflowing blood subsequently adhere to the initial layer of adherent platelets (platelet cohesion or aggregation) under flow have been less clearly defined.

The traditional model of platelet aggregation, in which integrin $\alpha_{\text {IIb }} \beta_{3}$ was thought to have an exclusive role in mediating platelet-platelet adhesion contacts, has been largely determined from studies using a platelet aggregometer (5). With this method, the addition of a soluble agonist to a stirred platelet suspension induces activation of integrin $\alpha_{\mathrm{II}} \beta_{3}$, converting it from a low- to a high-affinity receptor capable of binding soluble fibrinogen. The dimeric nature of fibrinogen enables it to cross-link adjacent activated platelets leading to stable platelet aggregation. Studies of platelet aggregation under high shear conditions, using a coneplate viscometer, have demonstrated that plasma vWf becomes the relevant ligand responsible for platelet activation (6). Shear-induced binding of soluble vWf to GPIb $\alpha$ initiates platelet activation independent of the addition of exogenous stimuli. Whereas the vWf-GPIb $\alpha$ interaction is indispensable for the initiation of platelet-platelet adhesion contacts under high shear, irreversible platelet aggregation requires a second adhesive interaction between $\mathrm{vWf}$ and integrin $\alpha_{\mathrm{II}} \beta_{3}$ (7).

The molecular events governing the formation of stable adhesion contacts between platelets in suspension have been well delineated; however, the mechanism by 
which platelets in free-flowing blood adhere to the surface of immobilized platelets has been less clearly defined. This is a critical issue, because platelet aggregation at sites of vascular injury does not generally occur between platelets in suspension, but rather involves the accrual of platelets from free-flowing blood onto the luminal surface of a developing thrombus. Thus, platelets immobilized at sites of vessel wall injury provide a highly reactive surface for the recruitment of additional platelets from flowing blood. Despite its fundamental importance for hemostasis and thrombogenesis, the nature of this reactive surface has remained incompletely understood.

In this study we have examined the mechanism by which platelets in flowing blood adhere to the surface of immobilized platelets using an in vitro flow-based aggregation assay and an in vivo rat thrombosis model. These studies demonstrated that platelet aggregation in vivo is a dynamic process in which platelets in flowing blood continuously tether, translocate, and/or detach from the luminal surface of a developing thrombus, with only a small percentage of tethering platelets forming stationary adhesion contacts. The tethering/translocation process is mediated by the binding of GPIb $\alpha$ on free-flowing platelets to $\mathrm{vWf}$ expressed on the surface of immobilized platelets. This tethering mechanism is indispensable for the formation of platelet adhesion contacts at arterial shear rates and also may enhance the efficiency of the platelet aggregation process at venous levels of shear.

\section{Methods}

Antibodies, constructs, and cell lines. Anti- $\alpha_{\mathrm{IIb}} \beta_{3} \mathrm{c7E} 3 \mathrm{Fab}$ was from Eli-Lilly (Centocor, Leiden, the Netherlands). Anti-GPIb $\alpha$ antibody, AN51, was from DAKO (Botany, New South Wales, Australia). Anti- $\alpha_{\text {IIb }} \beta_{3} F(a b)_{2}{ }^{\prime}$ RPM1, anti-GPIb $\alpha$ Fab RPM15, or the anti-GPV F(ab) ${ }_{2}{ }^{\prime}$ RPM9, against rat glycoproteins, were generated and characterized as reported previously (8). The anti-vWf A1 domain antibody, 5D2, was a generous gift from Michael Berndt (Baker Medical Research Institute, Melborne, Australia). The recombinant A1 domain was generated and characterized as reported elsewhere (9). Chinese hamster ovary $(\mathrm{CHO})$ cells were transfected with the GPIb/IX complex (CHOIb/IX) or vector alone (CHOpDX), as described previously (10).

In vitro flow studies. Blood was collected from healthy donors or individuals with Type III von Willebrand's disease (vWD; < $1 \%$ plasma and platelet vWf) or Glanzmann's thrombasthenia $\left(\mathrm{GT} ;<1 \% \alpha_{\mathrm{IIb}} \beta_{3}\right)$. Washed platelets were prepared from human donors (11) or Wistar rats as reported previously (12). Flow-based assays were performed according to a modified method of Cooke et al. (13). Confluent platelet monolayers (> 90\% surface coverage) were obtained by allowing washed platelets $\left(2 \times 10^{8} / \mathrm{mL}\right)$ to spread $\left(30-60\right.$ minutes, $\left.37^{\circ} \mathrm{C}\right)$ on glass microcapillary tubes (Vitro Dynamics Inc., Rockaway, New Jersey, USA). Microcapillaries were coated with type I equine tendon fibrillar collagen $(2.5 \mathrm{mg} / \mathrm{mL})$ overnight at $4^{\circ} \mathrm{C}$. Anticoagulated whole blood (15 mM trisodium citrate, $\mathrm{pH} 7.4)$ was incubated with the fluorescent dye $\mathrm{DiOC}_{6}(1 \mu \mathrm{M}, 10$ minutes; Molecular Probes Inc., Eugene, Oregon, USA), then perfused over preformed rat or human platelet monolayers at 150,600 , or $1800 \mathrm{~s}^{-1}$ for 1 minute. The majority of studies were performed with citrated blood, although similar findings were obtained with D-phenylalanyl-L-prolyl-L-arginine chloromethyl ketone-anticoagulated (PPACK-anticoagulated) blood $(40 \mu \mathrm{M})$. The interaction of flowing platelets with pre-formed platelet monolayers was viewed in real time using fluorescence microscopy and recorded on video for off-line analysis (see http://www.jci.org for supplementary data). In some experiments, human blood was preincubated for 10 minutes with c7E3 (20 $\mu \mathrm{g} / \mathrm{mL})$, AN51 $(0.6 \mu \mathrm{g} / \mathrm{mL})$, or the recombinant A1 domain of $\mathrm{vWf}(60 \mu \mathrm{g} / \mathrm{mL})$, whereas rat blood was preincubated for 10 minutes with the mAb RPM1 $(10 \mu \mathrm{g} / \mathrm{mL})$, RPM15 $(50 \mu \mathrm{g} / \mathrm{mL})$, or RPM9 $(50 \mu \mathrm{g} / \mathrm{mL})$.

Real-time analysis of platelet-platelet adhesion contacts. The number of platelets tethering to the surface of monolayers was analyzed frame by frame ( 50 frames per second) over the first 5-10 seconds of flow. In all studies, any cell forming an adhesion contact for more than 40 milliseconds was scored as a tethered platelet. Translocation was defined as platelet movement more than 1 cell diameter from the point of initial attachment. Stationary adhesion was defined as cells not moving more than a single cell diameter over a 10 -second time period.

Platelet-imaging studies. Immunofluorescence studies of human platelets were performed as described previously (11), using the 5D2 antibody $(0.76 \mu \mathrm{g} / \mathrm{mL})$. For scanning electron microscopy (SEM) studies, anticoagulated whole blood was perfused over spread platelet monolayers at $600 \mathrm{~s}^{-1}$ for 2 minutes. Adherent platelets were prepared for SEM as described previously (14). For the imaging of thrombus formation in real time (see Figure 3c), platelet-collagen interactions and subsequent thrombus growth was viewed using fluorescence confocal microscopy ( $\times 63$; Leica TCS SP; Leica, Heidelberg, Germany) and monitored at 1-minute intervals over a 5-minute time period. Thrombi were reconstructed in Voxblast (Vaytek Inc., Fairfield, Iowa, USA), and total thrombus volume and maximum height of thrombi in a given field (covering $25,192 \mu \mathrm{m}^{2}$ ) were calculated using ImageTool (University of Texas Health and Science Center of San Antonio, University of Texas, San Antonio, Texas, USA).

In vivo studies. In vivo rat thrombosis studies were performed according to a modified method of Denis et al. (15). Mesenteric arterioles and venules (30-40 $\mu \mathrm{m})$ of male Wistar rats were injured by photoactivation (excitation wavelength $550 \mathrm{~nm}, 10-30$ seconds) of systemically administered Rose Bengal (30 mg/kg) (16). The free radicals released from photoactivated Rose Bengal cause structural damage to the vessel wall resulting in the initiation of platelet thrombus formation (16). The dynamics of platelet interaction with the damaged vessel wall and with growing platelet thrombi were subsequently viewed using fluorescence or differential interference contrast 
(DIC) microscopy using a Leica DMIRB microscope $(\times 63$ 1.2NA lens) and recorded on video for off-line analysis. In some experiments, washed rat platelets were fluorescently labeled with $\mathrm{DiOC}_{6}$ and preincubated for 10 minutes with either buffer alone (control) or RPM1 $(10 \mu \mathrm{g} / \mathrm{mL})$, RPM15 $(50 \mu \mathrm{g} / \mathrm{mL})$, or RPM9 $(50 \mu \mathrm{g} / \mathrm{mL})$. Preincubated platelets were injected in the jugular vein, and their interaction with pre-formed arterial and venous thrombi were viewed using fluorescence microscopy. In vivo rat platelet survival upon pretreatment with the RPM antibodies was examined as described previously $(12,17)$. Shear rates in mesenteric vessels were determined by intravenous injection of fluorescent beads $(0.25 \mu \mathrm{m}$ diameter; Molecular Probes) through a jugular catheter (18). The velocities of beads in arterioles $(9-31.8 \mu \mathrm{m}$ diameter) and venules (26.9-90.1 $\mu \mathrm{m}$ diameter) were calculated according to Tangelder et al. (19). Shear rates were: arterioles, mean $1144 \pm 176 \mathrm{~s}^{-1}$ (range 580-1906, $n=3$ ); and venules, mean $343 \pm 47 \mathrm{~s}^{-1}$ (range 152-697, $n=3$ ).

\section{Results}

To investigate the mechanism of platelet aggregation as it occurs at sites of vascular injury, we established an in vitro flow-based aggregation assay, wherein fluorescently labeled platelets in whole blood were perfused over a nonlabeled spread platelet monolayer at venous $\left(150 \mathrm{~s}^{-1}\right)$, arterial $\left(600 \mathrm{~s}^{-1}\right)$, or arteriolar shear rates $\left(1800 \mathrm{~s}^{-1}\right)(20)$. As demonstrated in Figure 1a, spread platelet monolayers were able to support the adhesion of platelets from flowing blood, with the number of platelets adhering to the surface of the immobilized monolayer increasing as a function of shear. Analysis of the dynamics of individual platelet-platelet adhesion contacts demonstrated that the majority of platelets tethering to the surface of immobilized platelets subsequently translocated and/or detached (Figure $1 b)$. At low shear $\left(150 \mathrm{~s}^{-1}\right), 45 \pm 8 \%(n=6)$ of tethered platelets formed immediate stationary adhesion contacts (defined as cell movement $<1$ cell diameter from the point of initial contact over a 10-second time frame; Figure 1c), $36 \pm 5 \%$ of cells translocated (defined as cell movement $>1$ cell diameter from the point of initial contact), and $31 \pm 9 \%$ detached from the platelet monolayer (Figure 1b). With increasing shear, fewer platelets formed immediate stationary adhesion contacts $\left(9 \pm 4 \%\right.$ at $600 \mathrm{~s}^{-1}$ and $3 \pm 1 \%$ at $1800 \mathrm{~s}^{-1}$; Figure $\left.1 \mathrm{c}\right)$, with the majority of cells translocating $\left(63 \pm 4 \%\right.$ at $600 \mathrm{~s}^{-1}$ and $60 \pm 2 \%$ at $\left.1800 \mathrm{~s}^{-1}\right)$ and/or detaching from the platelet monolayer $(57 \pm 6 \%$ at $600 \mathrm{~s}^{-1}$ and $64 \pm 10 \%$ at $1800 \mathrm{~s}^{-1}$; Figure $\left.1 \mathrm{~b}\right)$. The dynamics of platelet-platelet interaction under flow were similar when using confluent platelet monolayers prepared on glass, immobilized vWf, or fibrinogen (data not shown), demonstrating that the phenomenon was not unique to glass-activated platelets. Moreover, platelet translocation and detachment was a prominent feature of thrombus growth, particularly at elevated shear rates (600 and 1800 $\mathrm{s}^{-1}$ ), when perfusing whole blood over pre-formed platelet thrombi (data not shown).

To examine the role of integrin $\alpha_{\mathrm{IIb}} \beta_{3}$ in mediating platelet aggregation under flow, whole blood was pre- treated with functional blocking antibodies against integrin $\alpha_{\mathrm{IIb}} \beta_{3}$ and perfused over a normal platelet monolayer. As demonstrated in Figure 1d, anti-integrin $\alpha_{\text {IIb }} \beta_{3}$ antibodies did not prevent platelet tethering or translocation on the platelet monolayer at 150,600 , or $1800 \mathrm{~s}^{-1}$, but inhibited the formation of stationary adhesion contacts by $97 \pm 2 \%$ at $150 \mathrm{~s}^{-1}, 98 \pm 1 \%$ at 600
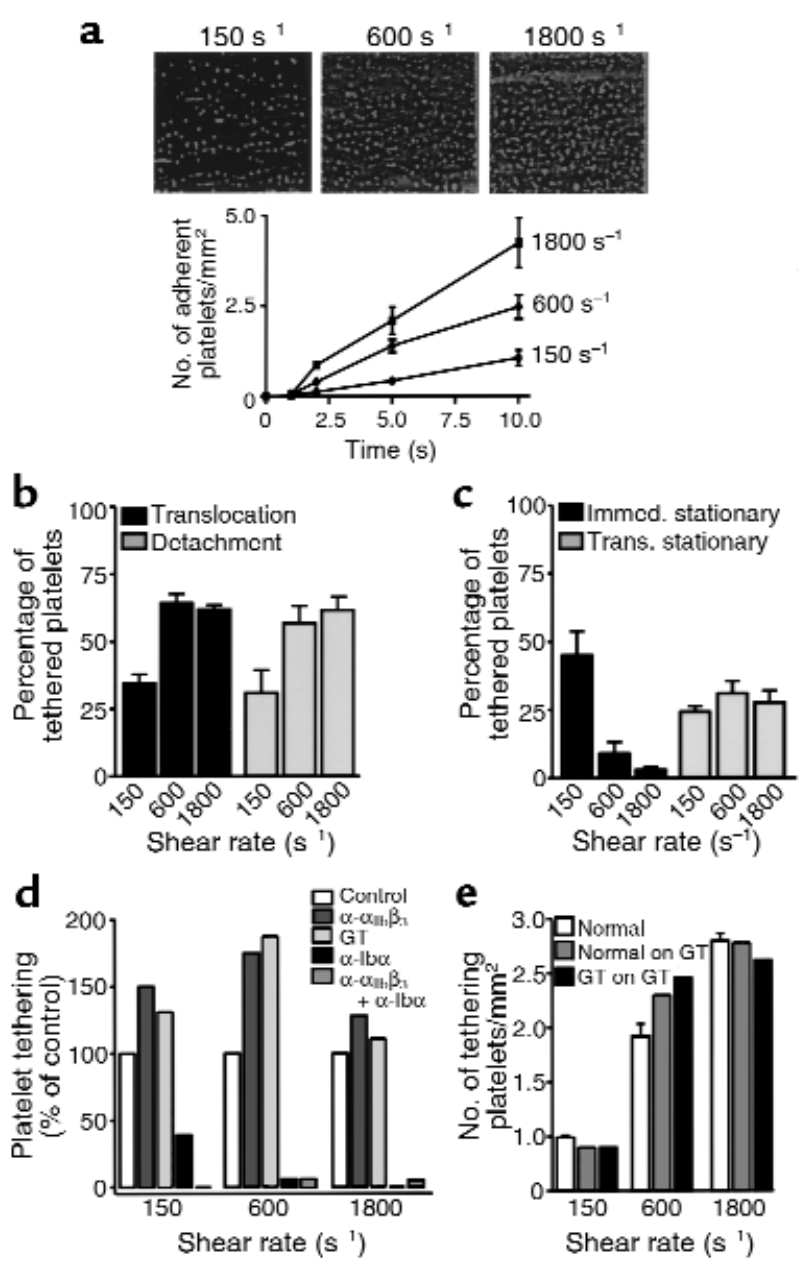

Figure 1

Dynamics of platelet aggregation under flow. Fluorescently labeled human platelets in whole blood were perfused over confluent spread platelet monolayers at 150,600 , and $1800 \mathrm{~s}^{-1}$ for 1 minute. (a) The images depict the shear-dependent increase in platelet adhesion after 1 minute of flow. The number of platelets tethering over a 10-second time period is demonstrated in the line graph. Each tethered platelet was examined over a further 10 -second time period, and the percentage of (b) platelets translocating and/or detaching and/or (c) forming stationary (immediate stationary or translocating then stationary) contacts was determined. Results represent mean $\pm \operatorname{SEM}(n=4)$. (d) Role of GPIb $\alpha$ in mediating platelet-platelet interactions under flow. Before perfusion, blood was incubated for 10 minutes with buffer (control), anti- $\alpha_{\| b} \beta_{3}\left(\alpha-\alpha_{\| 1 b} \beta_{3}\right)$, anti-GPIb $\alpha(\alpha-$-lb $\alpha)$, or both $\alpha-\alpha_{\| b} \beta_{3}$ and Ib $\alpha$ antibodies. In other studies GT platelets were perfused over normal platelet monolayers. (e) Fluorescently labeled platelets from a normal donor or a patient with GT were perfused over platelet monolayers prepared from a normal (normal) or a GT donor (normal on GT; GT on GT). The level of platelet tethering in the first 5 seconds of perfusion was determined. These results are representative of 3 experiments and are indicative of the tethering process over the full 1-minute perfusion period. 


\section{Figure 2}

Role of platelet vWf in mediating platelet tethering under flow. (a) Fluorescently labeled human platelets in whole blood from a normal donor were perfused over a platelet monolayer prepared from a normal donor (normal) or, alternatively, whole blood from an individual with vWD was perfused over a vWf-deficient platelet monolayer (vWD), in the presence or absence of an anti- $\alpha_{11 b} \beta_{3}\left(\alpha-\alpha_{11 b} \beta_{3}\right)$ antibody at the indicated shear rates. (b) Quantitation of platelet tethering in the first 5 seconds of perfusion. These results are representative of 5 experiments and are indicative of the tethering process over the full 1-minute perfusion period. (c) Platelet-platelet interactions occur at the site of vWf localization. Whole blood was perfused over a spread platelet monolayer at $600 \mathrm{~s}^{-1}$ for 2 minutes. Upper panels: These scanning electron micrographs demonstrate platelet tethering onto the granulomere of spread platelets (left image) and the early formation of a platelet aggregate (right image). These images are representative of over 100 cells examined. Lower panels: The images with white immunofluorescence ( $\alpha$-vWf; right half) partially overlaid onto the DIC image (left half) demonstrate that $\mathrm{vWf}$ becomes expressed on the surface of activated (spread) platelets and is specifically localized to the granulomere (right image). Note in the left image that $\mathrm{vWf}$ is not present on the surface of resting platelets. Bar, $5 \mu \mathrm{m}$.

\section{a}
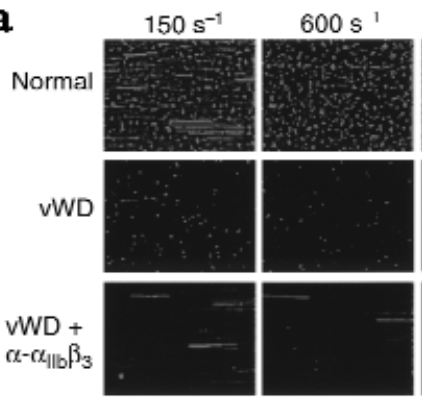

$1800 \mathrm{~s}^{-1}$

b

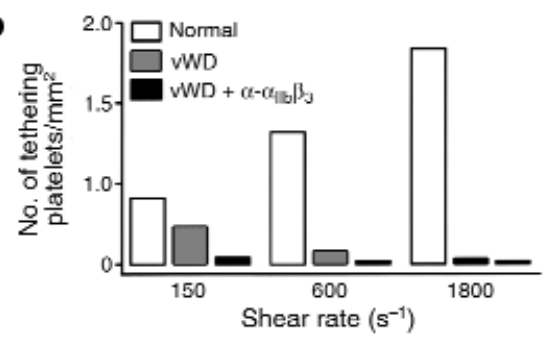

c

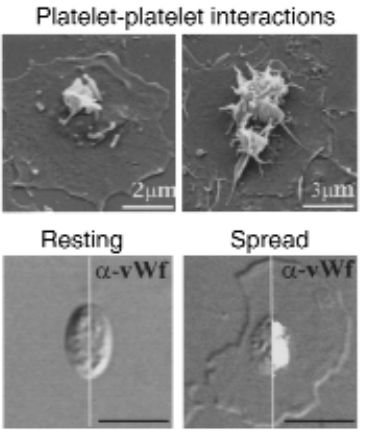

$\mathrm{s}^{-1}$, and $100 \%$ at $1800 \mathrm{~s}^{-1}$. Similar results were obtained using platelets congenitally deficient in $\alpha_{\mathrm{IIb}} \beta_{3}$ (GT; Figure $1 \mathrm{~d}$ ). It is interesting that in the absence of functional integrin $\alpha_{\mathrm{II}} \beta_{3}$ there was a paradoxical increase in the number of platelets tethering to immobilized platelets; however, the significance of this is not clear. To confirm that flowing platelets could tether to the surface of immobilized platelets in the complete absence of integrin $\alpha_{\mathrm{II}} \beta_{3}$, GT platelets in whole blood were perfused over immobilized GT platelets at 150,600 , or $1800 \mathrm{~s}^{-1}$. As demonstrated in Figure 1e, these cells effectively tethered and translocated at all shear rates examined but failed to form stationary adhesion contacts. Note that GT platelets spread poorly on glass, indicating that the tethering of flowing platelets to immobilized platelets is not dependent on platelet spreading.

To investigate the role of GPIb $\alpha$ in initiating platelet aggregation under flow, whole blood was treated with a blocking antibody against GPIb $\alpha$. As demonstrated in Figure 1d, blocking ligand binding to GPIb $\alpha$ on the surface of flowing platelets completely abolished platelet tethering at 600 and $1800 \mathrm{~s}^{-1}$ and resulted in approximately a $60 \%$ reduction in the number of tethered platelets at low shear $\left(150 \mathrm{~s}^{-1}\right)$. Concurrent blockade of both GPIb $\alpha$ and integrin $\alpha_{\mathrm{II}} \beta_{3}$ resulted in more than $95 \%$ reduction in platelet-platelet interaction under all shear conditions. These results suggest an indispensable role for GPIb $\alpha$ on the surface of flowing platelets in mediating platelet tethering at arterial shear rates and also indicate the involvement of this receptor in promoting platelet-platelet interactions at venous levels of shear.

To determine the relevant adhesive substrate on immobilized platelets responsible for platelet tethering, perfusion studies were performed on platelet monolayers prepared from individuals with severe Type III von Willebrand's disease $(<1 \% \mathrm{vWf})$. Perfusion of vWD whole blood over the vWf-deficient platelet monolayer under low shear conditions $\left(150 \mathrm{~s}^{-1}\right)$ resulted in approximately a 50\% reduction in platelet tethering relative to normal blood on a normal platelet monolayer (Figure 2 , a and b). At higher shear rates (600 and $1800 \mathrm{~s}^{-1}$ ), vWfdeficient platelets supported minimal platelet tethering $(<5 \%)$, and at all shear rates tested platelet translocation was not observed (data not shown). Together, these results suggest a key role for vWf on the surface of immobilized platelets and GPIb $\alpha$ on the surface of flowing platelets in mediating platelet tethering and translocation under flow. Three further independent lines of evidence confirmed that platelet tethering and translocation on the surface of immobilized platelets was mediated by the vWf-GPIb $\alpha$ interaction. First, GPIb/IX-transfected CHO cells, but not vector alone-transfected cells, tethered and translocated on immobilized normal platelets in a ristocetin-dependent manner, but failed to interact with vWD platelet mono- 
layers (data not shown). Second, the recombinant human vWf A1 domain completely abolished tethering of normal platelets under high shear conditions (data not shown). The inhibitory effect of the vWf A1 domain was specific to platelets because it had no effect on Pselectin-dependent leukocyte tethering and rolling on platelet monolayers. Third, analysis of more than 100 individual platelet-platelet adhesion contacts under flow demonstrated that all platelets tethered to the granulomere of immobilized platelets (Figure 2c, upper panel) at the site of vWf localization (Figure $2 c$, lower panel). Furthermore, all platelets translocated in a stopstart manner between granulomeres of immobilized platelets before forming irreversible adhesion contacts.

It has been shown that vWf is present in plasma as well as in the $\alpha$-granules and membranes of the surface connecting canalicular system of resting platelets (21). The relative ability of plasma and platelet vWf to support tethering of platelets in whole blood was investigated by perfusing vWD whole blood over a normal platelet monolayer, or conversely, by perfusing normal whole blood over a vWf-deficient platelet monolayer. As demonstrated in Figure 3a, normal platelet monolayers were highly effec- tive at supporting tethering of platelets from vWD blood at 150,600 , and $1800 \mathrm{~s}^{-1}$, despite the complete absence of plasma vWf. In contrast, vWf-deficient platelet monolayers supported only $20 \pm 3 \%$ and $23 \pm 4 \%$ platelet tethering compared with normal platelet monolayers at 600 and $1800 \mathrm{~s}^{-1}$, respectively (Figure 3a), despite normal levels of plasma vWf. Note that the data in Figure 3a was obtained during the earliest phase of the platelet aggregation process, i.e., in the first 5 seconds of perfusion. At this early time point sufficient plasma vWf may not have adsorbed on the surface of immobilized platelets. To investigate this possibility we examined the efficiency of platelet tethering over a 5 -minute time period and found that the reactivity of either normal or vWf-deficient platelet monolayers was maximal within the first 30 seconds of perfusion (data not shown). At the 30-second time point (Figure 3b), plasma $\mathrm{vWf}$ could restore platelet tethering by $69 \pm 21 \%(n=4)$ at $600 \mathrm{~s}^{-1}$ and by $35 \pm 8 \%(n=4)$ at $1800 \mathrm{~s}^{-1}$, relative to normal platelet monolayers. These findings suggest that plasma vWf can only partially compensate for the absence of platelet vWf in initiating platelet aggregation.

A potential limitation of our experimental approach was that the spread platelet monolayers were formed
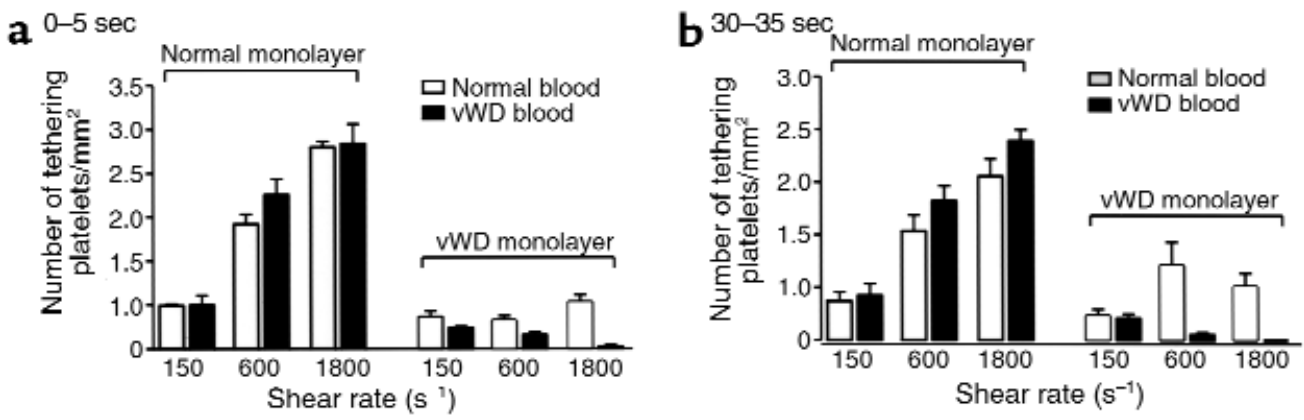

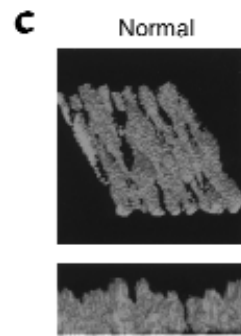

$22 \mu \mathrm{m}$

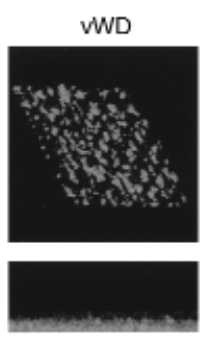

$4 \mu \mathrm{m}$

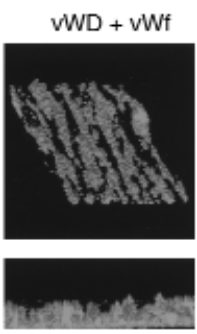

$13 \mu \mathrm{m}$ d

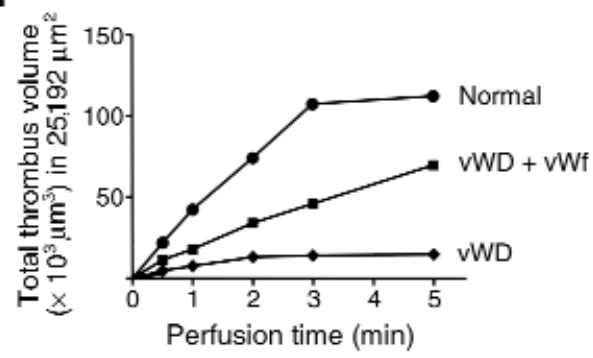

Figure 3

Relative roles of plasma and platelet vWf in initiating platelet aggregation under flow. Fluorescently labeled whole blood from a normal donor (normal blood) or a vWD patient (vWD blood) was perfused over pre-formed monolayers prepared with platelets from a normal donor (normal monolayer) or from a vWD patient (vWD monolayer) at the indicated shear rates. The level of platelet tethering in the first 5 seconds of flow (a) and the 5 seconds of flow after 30 seconds of perfusion (b) was determined. Results are presented as mean \pm SEM ( $n$ $=5)$. Note: The reduction in number of tethering platelets between 30-35 seconds relative to $0-5$ seconds reflects a decrease in the number of reactive sites available for platelet capture as a consequence of increased platelet adhesion. (c and $\mathbf{d}$ ) Role of platelet vWf in promoting thrombus growth at high shear. Fluorescently labeled whole blood from a normal donor or from a vWD patient was perfused over collagen at $150 \mathrm{~s}^{-1}$ for 2 minutes. This was followed by immediate perfusion of normal blood (normal), vWD blood (vWD), or vWD blood supplemented with purified soluble $\mathrm{vWf}(10 \mu \mathrm{g} / \mathrm{mL} ; \mathrm{vWD}+\mathrm{vWf})$ over the pre-formed reactive platelet surface at $1800 \mathrm{~s}^{-1}$. Thrombus growth was monitored in real time by confocal microscopy at 1-minute intervals for 5 minutes. (c) Platelet thrombi formed after 5 minutes of perfusion were reconstructed using a computer-assisted image-analysis program. The upper panels represent an oblique view to demonstrate surface coverage while the lower panels represent a side-on view to demonstrate differences in thrombus height. (d) Total thrombus volume in a field of interest $\left(25,192 \mu \mathrm{m}^{2}\right)$ was calculated using Image Tool. Note: When calculating thrombus volumes, the first $2 \mu \mathrm{m}$ were omitted from analysis to exclude the pre-formed platelet monolayers. The results are from 1 experiment representative of 2 . 


\section{Figure 4}

Dynamics of rat platelet aggregation in vitro and in vivo. Fluorescently labeled rat platelets in whole blood were perfused over spread rat platelet monolayers at 150, 600, and $1800 \mathrm{~s}^{-1}$ for 1 minute. (a) The percentage of tethered platelets translocating and/or detaching over a 10-second period is demonstrated. (b) Before perfusion, blood was incubated for 10 minutes with buffer (control), anti- $\alpha_{I ! b} \beta_{3}\left(\alpha-\alpha_{I ! b} \beta_{3}\right)$, antiGPV $(\alpha-G P V)$, or anti-GPIb $\alpha(\alpha-I b \alpha)$ antibody fragments. In these studies the number of platelets tethering over a 5-second time period is demonstrated. Results represent mean $\pm \operatorname{SEM}(n=4)$. (c) Dynamics of platelet aggregation in vivo. Vascular injury and thrombus formation in rat mesenteric vessels was induced by photoactivation of Rose Bengal. The percentage of platelets translocating and/or detaching from the surface of arterial and venous thrombi was examined over a 10 -second time period using DIC microscopy. (d) Following thrombus formation, fluorescently labeled, washed rat platelets, preincubated with buffer (control), anti-GPIb $\alpha(\alpha-G P I b \alpha)$, anti- $\alpha_{\| 1} \beta_{3}\left(\alpha-\alpha_{\| 1} \beta_{3}\right)$, or anti-GPV ( $\alpha$-GPV) antibody fragments were injected intravenously. Pre-formed platelet thrombi (white arrowheads) were viewed by differential interference contrast (upper panels; DIC) whereas tethering of injected platelets to the surface of preformed thrombi was observed by fluorescence microscopy (lower panels; $\times 63$ ). For clarity, the mesenteric arteriole walls have been demarcated using continuous lines, whereas the surface of thrombi have been outlined using discontinuous lines. Uninjured refers to a mesenteric vessel that has not been subjected to photo-induced injury. These images are from 5 experiments and are representative of more than 50 independent experiments. Note the $\alpha$ - $\alpha_{11} \beta_{3}$-treated platelets that adhered to the surface of arterial thrombi formed transient tethers. All these cells subsequently translocated and/or detached from the thrombus. under static assay conditions in the absence of plasma fibrinogen or vWf, conditions that may preferentially favor the adhesive function of platelet vWf. To investigate further the relative role of platelet and plasma vWf in promoting platelet aggregation under more physiological conditions, a 2-step flow assay was established. In these studies, normal blood was initially perfused over type 1 fibrillar collagen at low shear $\left(150 \mathrm{~s}^{-1}\right)$ for 2 minutes to allow platelet coverage of the reactive surface. This was immediately followed by perfusion of normal blood, vWD blood, or vWD blood supplemented with purified vWf $(10 \mu \mathrm{g} / \mathrm{mL})$ over the adherent platelets at a wall shear rate of 1800 $\mathrm{s}^{-1}$, and the subsequent growth in thrombi was monitored by confocal imaging. As demonstrated in Figure 3, c and d, perfusion of normal blood over the reactive platelet surface resulted in a time-dependent increase in thrombus volume. In contrast, vWD blood formed small thrombi under these conditions. Addition of purified vWf $(10 \mu \mathrm{g} / \mathrm{mL})$ to the $\mathrm{vWD}$ blood partially restored the rate and extent of thrombus growth, albeit to approximately half the level observed in normal blood (maximal thrombus height of $22 \mu \mathrm{m}$ for normal blood versus $13 \mu \mathrm{m}$ for vWD blood containing purified $v W f)$. Addition of higher concentrations of purified vWf $(20-40 \mu \mathrm{g} / \mathrm{mL})$ to $\mathrm{vWD}$ blood failed to restore normal thrombus growth (data not shown), demonstrating that supraphysiological concentrations of plasma vWf do not fully compensate for the loss of platelet vWf.

To investigate the physiological significance of our in vitro findings, we examined the dynamics of platelet aggregation in vivo using a rat thrombosis model (15). We initially confirmed that the dynamics of rat platelet aggregation in vitro were similar to what we had observed using human platelets. As demonstrated in Figure $4 a$, the majority of rat platelets tethering to the surface of a spread rat platelet monolayer subsequently translocated and/or detached at all shear rates examined. Moreover, pretreating whole blood with a blocking antibody Fab fragment against GPIb $\alpha$ or an $\mathrm{F}(\mathrm{ab})_{2}{ }^{\prime}$ fragment against integrin $\alpha_{\text {IIb }} \beta_{3}$ yielded results similar to that observed with human platelets (Figure 4b). To investigate the dynamics of platelet aggregation in vivo, we performed intravital microscopy on injured rat mesenteric arterioles and venules, according to a modified method of Denis et al. (15). Analysis of the early stages of platelet thrombus formation in rat arterioles using high-magnification DIC microscopy demonstrated adhesion of platelets to the vessel wall within 1-2 minutes of photoactivation followed by platelet accrual onto the developing thrombus. Interestingly, more than $95 \%$ of platelets tethering to the luminal surface of growing thrombi in both arterioles and 
venules subsequently translocated and/or detached. The dynamics of platelet aggregation were consistent in all vessels examined $(n=100)$ and at all stages of thrombus growth. Moreover, there was a strong correlation between the dynamics of platelet aggregation in vivo to that observed in vitro (Figure $4 \mathrm{c}$ ). To investigate the role of GPIb $\alpha$ and integrin $\alpha_{\mathrm{IIb}} \beta_{3}$ in mediating platelet tethering to pre-formed thrombi in vivo (Figure $4 \mathrm{~d}$, upper panels), fluorescently labeled washed rat platelets were pretreated with the anti-GPIb $\alpha$ and integrin $\alpha_{\mathrm{IIb}} \beta_{3} \mathrm{Fab}$ and $\mathrm{F}(\mathrm{ab})_{2}{ }^{\prime}$ fragments, respectively, before intravenous injection. As observed in the in vitro flow experiments, blocking ligand binding to integrin $\alpha_{\text {IIb }} \beta_{3}$ did not appear to markedly reduce the number of platelets tethering to the surface of pre-formed thrombi. In contrast, blocking ligand binding to GPIb $\alpha$ completely abolished the ability of platelets to tether to the luminal surface of pre-formed thrombi in arterioles (Figure 4d, lower middle panel), and reduced platelet tethering to venous thrombi (data not shown). As observed in vitro, platelets pretreated with the antiGPIb $\alpha$ antibody that tethered to venous thrombi failed to translocate, confirming that this process is a GPIb $\alpha$ dependent phenomenon. In control studies, $\mathrm{F}(\mathrm{ab})_{2}{ }^{\prime}$ fragments against GPV did not appear to affect platelet-platelet interactions in either the arterial or venous circulation. Additionally, we confirmed that the reduced interaction of anti-GPIb $\alpha$-treated platelets with thrombi was not an artifact of platelet sequestra- tion because the majority of the infused platelets $(\sim 63 \%)$ remained circulating 30 minutes after injection (data not shown).

\section{Discussion}

The studies presented here demonstrate an unexpected complexity to the platelet aggregation process in vivo, in which the majority of platelets tethering to the luminal surface of a developing thrombus subsequently translocate and/or detach, with only a small percentage of tethered platelets forming stationary adhesion contacts at both arterial and venous shear rates. Moreover, we demonstrate an important role for platelet $\mathrm{vWf}$ in mediating platelet tethering and translocation through a specific interaction with GPIb $\alpha$. The vWf-GPIb $\alpha$ interaction is indispensable for platelet tethering at arterial shear rates and also appears to promote platelet aggregation at venous levels of shear.

Our studies demonstrate an important role for vWf in recruiting platelets from flowing blood onto the luminal surface of a developing thrombus (see Figure 5). Several lines of evidence suggest that this tethering mechanism is not only operative at arterial flow rates but also at venous levels of shear. First, pretreating freeflowing platelets with the anti-GPIb $\alpha$ antibody reduced platelet tethering by approximately $60 \%$ at 150 $\mathrm{s}^{-1}$. Second, monolayers prepared from $\mathrm{vWD}$ platelets supported approximately 50\% less tethering than monolayers prepared from normal platelets. Third,

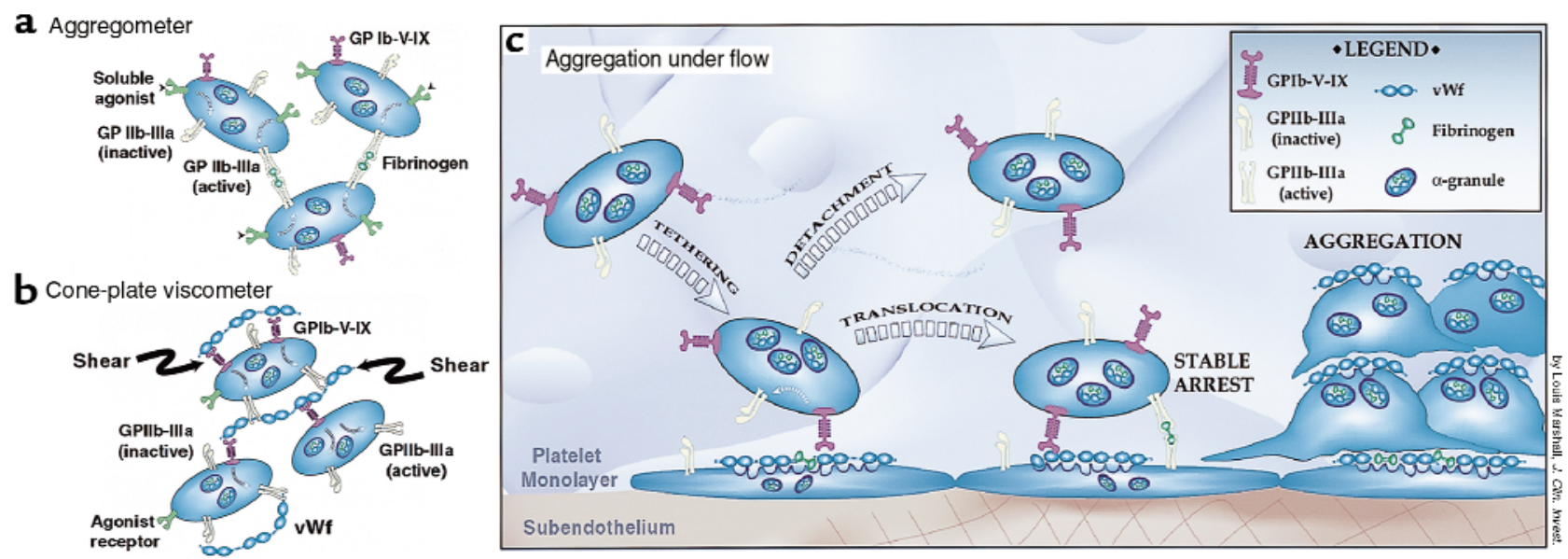

Figure 5

A revised model of platelet aggregation. ( $\mathbf{a}$ and $\mathbf{b}$ ) The mechanisms by which platelets in suspension aggregate following exposure to a soluble agonist or high shear stress are depicted. (c) The multistep mechanism by which circulating platelets form stationary adhesion contacts with the luminal surface of a growing thrombus under physiological flow conditions is demonstrated. (a) The addition of a soluble agonist to a stirred platelet suspension leads to the activation of integrin $\alpha_{11 b} \beta_{3}$ (GPIIb-IIla) and its subsequent binding to soluble fibrinogen. Fibrinogen's dimeric structure enables it to cross-link adjacent activated platelets and mediate stable platelet aggregation (5, 38). (b) Under high shear conditions, vWf becomes the relevant ligand responsible for platelet activation. Shear-induced binding of soluble vWf to GPIb$\mathrm{V}$-IX induces platelet activation. At elevated levels of shear, stable platelet aggregation is thought to be dependent primarily on $v W f$ binding to GPIIb-IIla $(7,39)$. (c) Upon vascular injury, platelets are recruited to the subendothelium where they become activated, releasing their granule contents. vWf expressed on the surface of immobilized platelets tethers circulating platelets by binding GPIb $\alpha$. The majority of platelets that tether subsequently translocate or detach from the surface of immobilized platelets. The formation of stationary adhesion contacts is dependent on the cross-linking of integrin $\alpha_{11} \beta_{3}$ on the surface of free-flowing and immobilized platelets by plasma vWf or fibrinogen. This multistep adhesion process operates at both arterial and venous levels of shear and appears to be the predominant mechanism by which platelets aggregate in vivo. However, note that at venous levels of shear, platelets can also form immediate stationary adhesion contacts with immobilized platelets independent of the vWf-GPIb $\alpha$ interaction. 
approximately $40 \%$ of rat and human platelets translocated on immobilized platelets at low shear rates. Fourth, normal platelets tethered and translocated on a GT platelet monolayer in a GPIb $\alpha$-dependent manner at venous shear rates. Fifth, platelet translocation at all shear rates was completely abolished by pretreating whole blood with the anti-GPIb $\alpha$ antibody and was not observed on a vWD platelet monolayer. Finally, pretreating rat platelets with an anti-GPIb $\alpha$ antibody reduced their ability to tether to pre-formed venous thrombi in vivo. These observations are consistent with two previous reports suggesting that the vWf-GPIb $\alpha$ interaction may participate in adhesion processes at venous shear rates $(22,23)$.

The involvement of the vWf-GPIb $\alpha$ interaction in promoting platelet aggregation at low shear appears to contradict previous findings demonstrating that thrombus growth on type 1 fibrillar collagen under low shear conditions occurs independent of both vWf and GPIb $\alpha$. $(23,24)$. A possible explanation for this apparent paradox requires consideration of the relevant roles of GPIb/IX and integrin $\alpha_{\text {IIb }} \beta_{3}$ in promoting thrombus growth under different shear conditions. The importance of GPIb/IX in initiating platelet aggregation at high shear is consistent with the unique biomechanical properties of the vWf-GPIb $\alpha$ bond that enables efficient capture of platelets from rapidly flowing blood. In contrast, ligand binding to integrin $\alpha_{\mathrm{II}} \beta_{3}$ is characterized by an intrinsically slow on-off rate, a characteristic that is ideal for sustaining platelet adhesion contacts but less optimal for initiating platelet-surface interactions, especially in high flow situations. It is therefore not surprising that the vWf-GPIb $\alpha$ interaction may facilitate the initial interaction of platelets with a growing thrombus even at low shear rates. However, the critical determinant of thrombus growth is not the number of platelets tethering to the surface of a growing thrombus, but the number of tethering cells forming stable adhesion contacts, primarily a reflection of the activation status of integrin $\alpha_{\text {IIb }} \beta_{3}$. Thus a potent thrombogenic surface such as type 1 fibrillar collagen, which readily induces integrin $\alpha_{\text {IIb }} \beta_{3}$ activation and thereby increases the probability of a tethered platelet forming an irreversible adhesion contact, is likely to rapidly induce platelet aggregation independent of vWf and GPIb $\alpha$. It remains to be established, however, whether other adhesive substrates, such as collagen type VI, which tethers platelets inefficiently under shear conditions as low as $100 \mathrm{~s}^{-1}$ (23), require the synergistic contribution of GPIb/IX and other adhesion receptors to promote thrombus growth at venous shear rates.

A key finding in this study is the important role of platelet vWf in initiating platelet aggregation under flow. Platelet vWf represents between $8 \%(25)$ and $15 \%$ (26) of the total circulating $v W f$ and is stored in the $\alpha$-granules and membranes of the surface-connecting canalicular system (21). Previous studies have suggested that $60-70 \%$ of released platelet vWf binds to the surface of platelets in an integrin $\alpha_{\mathrm{IIb}} \beta_{3}$-dependent manner (27).
Our studies using Glanzmann platelet monolayers demonstrate that functionally relevant levels of platelet vWf can be expressed on the platelet surface independent of integrin $\alpha_{\mathrm{IIb}} \beta_{3}$. Whereas these findings do not exclude a potentially important role for integrin $\alpha_{\mathrm{IIb}} \beta_{3}$ in enhancing surface expression of platelet vWf, they point to the existence of additional binding sites on the cell surface. To date, a role for GPIb $\alpha$ in localizing platelet vWf to the cell surface has not been established. Platelet vWf binds to GPIb $\alpha$ with a significantly lower affinity than the plasma protein despite the fact that platelet vWf consists of higher-molecular-weight multimers (28) and that these multimers are preferentially expressed on the cell surface $(29,30)$. Whether GPIb $\alpha$ can functionally compensate in the absence of integrin $\alpha_{\mathrm{IIb}} \beta_{3}$ or an additional receptor is involved in vWf surface expression is an important issue for future investigation.

Our findings that platelet vWf plays a key role in initiating platelet aggregation under flow are in keeping with previous clinical and experimental studies suggesting an important hemostatic function for this pool of $v W f$ (see review by Mannucci [31]). For example, it is well established that the level of platelet vWf acts as a major determinant of the skin bleeding time in healthy individuals and in Type $1 \mathrm{vWD}(25,32)$. Furthermore, correction of the hemostatic defect in Type III vWD requires replacement of both the plasma and platelet pools of vWf (33). Additional evidence for a role for platelet vWf in hemostasis stems from studies of vWD pigs following transplantation with normal bone marrow. The bleeding times in these pigs, expressing normal levels of platelet vWf, was significantly shortened despite low levels of the plasma protein (34). Moreover, in ex vivo perfusion experiments these platelets adhere poorly to fibrillar collagen and have impaired thrombus formation at elevated shear rates $(35,36)$. It has generally been assumed that this reduction in thrombus growth is primarily a reflection of defective platelet adhesion rather than impaired aggregation. By analyzing thrombus growth on a preformed platelet surface, thereby eliminating the contribution of defective platelet-substratum interactions to thrombus growth, we have highlighted the important contribution of platelet vWf to the aggregation process.

Our findings suggest an important synergistic relationship between the plasma and platelet pools of vWf in supporting platelet aggregation under flow. The inability of plasma vWf to fully compensate for deficiency of platelet vWf in our flow experiments would explain why an isolated deficiency of platelet vWf leads to a prolonged bleeding time and why replacement of plasma vWf in severe vWD only partially corrects the hemostatic defect (33). The reason(s) why plasma vWf does not fully compensate for loss of the platelet protein remains unclear. Differences in the multimer composition of plasma and platelet vWf (28), the ability of plasma fibrinogen to effectively compete with plasma vWf for binding sites on the surface of immobilized platelets (37), and the possibility of distinct binding sites for the platelet protein may partly explain the functional differ- 
ences between these two proteins. A key issue for future investigation will be to determine the relative contribution of the platelet and plasma pools of both vWf and fibrinogen in supporting the various stages of platelet thrombus growth and to delineate the mechanisms regulating surface immobilization of these individual adhesive substrates. Unraveling the mechanism(s) regulating vWf surface expression on platelets will not only be important for an improved understanding of the hemostatic and thrombotic processes, but may also allow the development of novel therapeutic strategies aimed at reducing platelet thrombus formation in vivo.

\section{Acknowledgments}

We would like to thank Hatem Salem for his constructive criticisms and helpful advice throughout this study; Louis Marshall for assistance with imaging and graphics; Sylvie Moog, Simone Schuhler, Yuping Yuan, Nayna Mistry, and Susan L. Cranmer for technical assistance; and Michael Berndt for his generous donation of anti-vWf antibodies. We would especially like to thank the individuals with von Willebrand's disease and Glanzmann's thrombasthenia for their generous donations of blood. This work was supported by grants from the National Health and Medical Research Council of Australia and the National Heart Foundation of Australia. S. Kulkarni is a recipient of an Australian Post-Graduate Award.

1. Savage, B., Almus-Jacobs, F., and Ruggeri, Z.M. 1998. Specific synergy of multiple substrate-receptor interactions in platelet thrombus formation under flow. Cell. 94:657-666.

2. Tschopp, T.B., Weiss, H.J., and Baumgartner, H.R. 1973. Decreased adhesion of platelet to subendothelium in von Willebrand's disease. J. Lab. Clin. Med. 83:296-300

3. Weiss, H.J., Turitto, V.T., and Baumgartner, H.R. 1978. Effect of shear rate on platelet interaction with subendothelium in citrated and native blood. I. Shear rate-dependent decrease of adhesion in von Willebrand's disease and the Bernard-Soulier syndrome. J. Lab. Clin. Med. 92:750-764.

4. Savage, B., Saldivar, E., and Ruggeri, Z.M. 1996. Initiation of platelet adhesion by arrest onto fibrinogen and translocation on von Willebrand factor. Cell. 84:289-297.

5. Holmsen, H. 1986. Platelet responses and metabolism. CRC Press. Boca Raton, FL. Pp 11.

6. Goto, S., Ikeda, Y., Saldivar, E., and Ruggeri, Z.M. 1998. Distinct mechanisms of platelet aggregation as a consequence of different shearing flow conditions. J. Clin. Invest. 101:479-486.

7. Ruggeri, Z.M. 1993. Mechanisms of shear-induced platelet adhesion and aggregation. Thromb. Haemost. 70:119-123.

8. Ravanat, C., et al. 1997. Gene cloning of rat and mouse platelet glycoprotein $\mathrm{V}$ : identification of megakaryocyte-specific promoters and demonstration of functional thrombin cleavage. Blood. 89:3253-3262.

9. Prior, C.P., et al. 1993. Optimization of a recombinant von Willebrand factor fragment as an antagonist of the platelet glycoprotein Ib receptor. Biotechnology. 11:709-713.

10. Cranmer, S.L., et al. 1999. Glycoprotein (GP) Ib-IX-transfected cells roll on a von Willebrand factor matrix under flow: importance of the GPIb/actin-binding protein (ABP-280) interaction in maintaining adhesion under high shear. J. Biol. Chem. 274:6097-6106.

11. Yuan, Y., Dopheide, S.M., Ivanidis, C., Salem, H.H., and Jackson, S.P. 1997. Calpain regulation of cytoskeletal signalling complexes in von Willebrand factor-stimulated platelets. J. Biol. Chem. 272:21847-21854.

12. Freund, M., et al. 1993. Experimental thrombosis on a collagen coated arterioarterial shunt in rats: a pharmacological model to study antithrombotic agents inhibiting thrombin formation and platelet deposition. Thromb. Haemost. 69:515-521.
13. Cooke, B.M., Usami, S., Perry, I., and Nash, G.B. 1993. A simplified method for culture of endothelial cells and analysis of adhesion of blood cells under conditions of flow. Microvasc. Res. 45:33-55.

14. Schoenwaelder, S.M., et al. 1994. Tyrosine kinases regulate the cytoskeletal attachment of integrin $\alpha_{\mathrm{IIb}} \beta_{3}$ (platelet glycoprotein IIb/IIIa) and the cellular retraction of fibrin polymers. J. Biol. Chem. 51:32479-32487.

15. Denis, C., et al. 1998. A mouse model of severe von Willebrand disease: defects in haemostasis and thrombosis. Proc. Natl. Acad. Sci. USA. 95:9524-9529.

16. Saniabaoi, A.R., Umemura, K., Matsumoto, N., Sakuma, S., and Nakashima, M. 1995. Vessel wall injury and arterial thrombosis induced by a photochemical reaction. Thromb. Haemost. 73:868-872.

17. Ravanat, C., et al. 1999. Differential effects of monoclonal antibodies to rat GPIIb-IIIa, GPIb $\alpha$, or GPV on platelet function and in vivo survival. Thromb. Haemost. 82(Suppl.):528.

18. Yale, C.E., and Torhost, J.B. 1972. Critical bleeding and plasma volumes of the adult Gerfree rat. Lab. Anim. Sci. 22:497-502.

19. Tangelder, G.J., Slaaf, D.W., Arts, T., and Reneman, R.S. 1988. Wall shear rate in arterioles in vivo: least estimates from platelet velocity profiles. Am. J. Physiol. 254:H1059-H1064.

20. Kroll, M.H., Hellums, D., McIntire, L.V., Schafer, A.I., and Moake, J.L. 1996. Platelets and shear stress. Blood. 88:1525-1541.

21. Rand, J.H., Gordon, R.E., Uson, R.R., and Potter, B.J. 1987. Localization of surface vWf on resting and stimulated platelets. Blood. 70:1297-1302.

22. Badimon, L., Badimon, J.J., Turitto, V.T., and Fuster, V. 1989. Role of von Willebrand factor in mediating platelet-vessel wall interaction at low shear rate: the importance of perfusion conditions. Blood. 73:961-967.

23. Mazzucato, M., et al. 1999. Identification of domains responsible for von Willebrand factor Type VI collagen interaction mediating platelet adhesion under high flow. J. Biol. Chem. 274:3033-3041.

24. Andre, P., et al. 1998. Role of plasma and platelet von Willebrand factor in arterial thrombogenesis and hemostasis in the pig. Exp. Hematol. 26:620-626

25. Rodeghiero, F., Castaman, G., Ruggeri, M., and Tosetto, A. 1992. The bleeding time in normal subjects is mainly determined by platelet von Willebrand factor and is independent from blood group. Thromb. Res. 65:605-612.

26. Zucker, M.B., Broekman, M.J., and Kaplan, K.L. 1979. Factor VII-related antigen in human blood platelets: localization and release by thrombin and collagen. J. Lab. Clin. Med. 94:675-682.

27. Parker, R.I., and Gralnick, H.R. 1986. Identification of platelet glycoprotein IIb/IIIa as the major binding site for released platelet von-Willebrand factor. Blood. 68:732-736.

28. Williams, S.B., McKeown, L.P., Krutzsch, H., Hansmann, K., and Gralnick, H.R. 1994. Purification and characterisation of human platelet von Willebrand factor. Br. J. Haematol. 88:582-591.

29. George, J.N., and Onofre, A.R. 1982. Human platelet surface binding of endogenous secreted factor VIII-von Willebrand factor and platelet factor 4. Blood. 59:194-197.

30. Fernandez, M.F.L., Ginsberg, M.H., Ruggeri, Z.M., Batlle, F.J., and Zimmerman, T.S. 1982. Multimeric structure of platelet factor VIII/von Willebrand factor: the presence of large multimers and their reassociation with thrombin-stimulated platelets. Blood. 60:1132-1138.

31. Mannucci, P.M. 1995. Platelet von Willebrand factor in inherited and acquired bleeding disorders. Proc. Natl. Acad. Sci. USA. 92:2428-2432.

32. Gralnick, H.R., et al. 1986. Platelet von Willebrand factor: an important determinant of the bleeding time in Type I von Willebrand's disease. Blood. 68:58-61.

33. Castillo, R., et al. 1991. Haemostatic effect of normal platelet transfusion in severe von Willebrand disease patients. Blood. 77:1901-1905.

34. Bowie, E.J., et al. 1986. Transplantation of normal bone marrow into a pig with severe von Willebrand's disease. J. Clin. Invest. 78:26-30.

35. Nichols, T.C., et al. 1995. Function of von Willebrand factor after crossed bone marrow transplantation between normal and von Willebrand disease pigs: effect on arterial thrombosis in chimeras. Proc. Natl. Acad. Sci. USA. 92:2455-2459.

36. Fressinaud, E., Baruch, D., Rothschild, C., Baumgartner, H.R., and Meyer, D. 1987. Platelet von Willebrand factor: evidence for its involvement in platelet adhesion to collagen. Blood. 70:1214-1217.

37. Gralnick, H.R., Williams, S.B., and Coller, B.S. 1984. Fibrinogen competes with von Willebrand factor for binding to the glycoprotein IIb/IIIa complex when platelets are stimulated with thrombin. Blood. 64:797-800.

38. Clemetson, K.J. 1999. Primary haemostasis: sticky fingers cement the relationship. Curr. Biol. 9:R110-R112.

39. Ikeda, Y., et al. 1991. The role of von Willebrand factor and fibrinogen in platelet aggregation under varying shear stress. J. Clin. Invest. 87:1234-1240. 